\title{
Black holes: from stars to galaxies
}

\author{
I. Félix Mirabel $\dagger$ \\ European Southern Observatory, Alonso de Cordova 3107, Santiago, Chile \\ email: fmirabel@eso.org
}

\begin{abstract}
While until recently they were often considered as exotic objects of dubious existence, in the last decades there have been overwhelming observational evidences for the presence of stellar mass black holes in binary systems, supermassive black holes at the centers of galaxies, and possibly, intermediate-mass black holes observed as ultraluminous X-ray sources in nearby galaxies. Black holes are now widely accepted as real physical entities that play an important role in several areas of modern astrophysics.

Here I review the concluding remarks of the IAU Symposium No 238 on Black Holes, with particular emphasis on the topical questions in this area of research.
\end{abstract}

Keywords. Black hole physics - galaxies: nuclei - galaxies: jets - stars: general

\section{The aim of IAU Symposium No 238}

The interaction of black holes with their surroundings produces analogous phenomena in AGN and stellar black hole binaries. The scales of length and time of the phenomena are proportional to the mass of the black hole, and the whole phenomenological diversity that takes place around black holes can be described by the same physical concepts, however, the observed phenomena exhibit enormous complexity (see figure 1). Quasars and microquasars can eject matter several times, whereas collapsars form jets only once. When the jets point to the Earth these objects appear as microblazars, blazars and gamma-ray bursts, respectively. Synergy between research on stellar mass and supermassive black holes has become essential for our understanding of the underlying physics.

With this in mind, the aim of IAU Symposium No 238 has been to discuss the relations between different types of astrophysical black holes in a broader evolutionary context, bringing together astronomers working on AGN with those working on compact stellar binaries. Several groups that actively search for the elusive intermediate-mass black holes have had an active participation.

\section{Stellar black holes}

Current physics suggests that compact objects in stellar binaries with mass functions larger than 4 solar masses must be black holes. There are about 20 known objects with such mass functions. They are believed to be the tip of an iceberg, since it is estimated that in the Milky Way alone there should be at least 1000 dormant black hole X-ray transients, while the total number of stellar-mass black holes (isolated and in binaries) could be as large as 100 million. The number statistics of known stellar black holes is still very small and at present remain open the following questions:

Is the gap in the black hole mass function between 2.2 and 4.0 solar masses real? If so, which is the physical reason? Why in the Milky Way it has not been found a stellar

$\dagger$ On leave from CEA, France. 


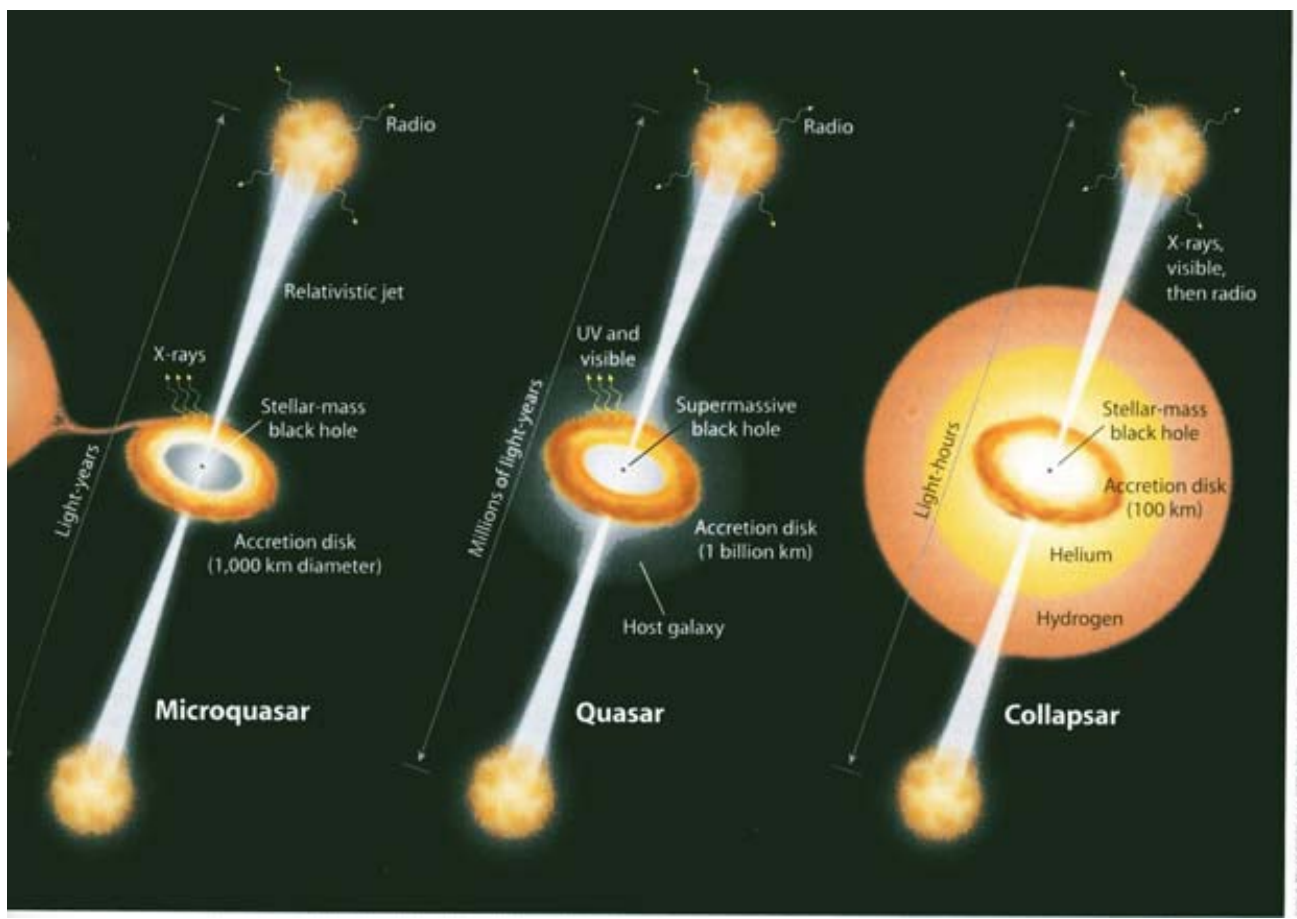

Figure 1. The same physical mechanism can be responsible for the three different types of objects: microquasars, quasars/AGN and massive stars that collapse ("collapsars") to form a black hole producing Gamma-Ray-Bursts. Each one of these objects contains a black hole, an accretion disk and relativistic particles jets. Credit: Mirabel \& Rodríguez (2002).

black hole with mass larger than 14 solar masses? Is this due to poor statistics or to large mass losses in stellar winds by the metal rich progenitors in the Milky Way?

Kinematic studies of black hole binaries suggest that some stellar black holes form associated to very energetic supernova explosions (see figure 2). In some cases, this kinematic evidence is reinforced by the chemical composition of the donor star, when it contains elements produced in supernova explosions. However, kinematic studies suggest that black holes may also form by direct collapse, namely, in the dark (figure 3 ). Therefore, it is an open question when stellar black holes form in energetic supernovae and when by direct core collapse; more specifically, whether the presence of an energetic explosion depends or not on the mass of the collapsing stellar core. In fact, the kinematics of the microquasars Cygnus X-1 and GRS 1915+105, which contain black holes of $\sim 10$ and $\sim 14$ solar masses, respectively, did not receive strong kicks from natal energetic supernova explosions.

This question on the explosive or implosive black hole formation can be approached by observations of nearby gamma-ray bursts of long duration, which are believed to take place when stellar black holes are form by core collapse of massive stars. Therefore, the question on whether all collapsar GRBs are associated with supernovae of type Ia/b, or some are not, is of topical interest for the understanding of the very last phases of massive stellar evolution and black hole formation. 

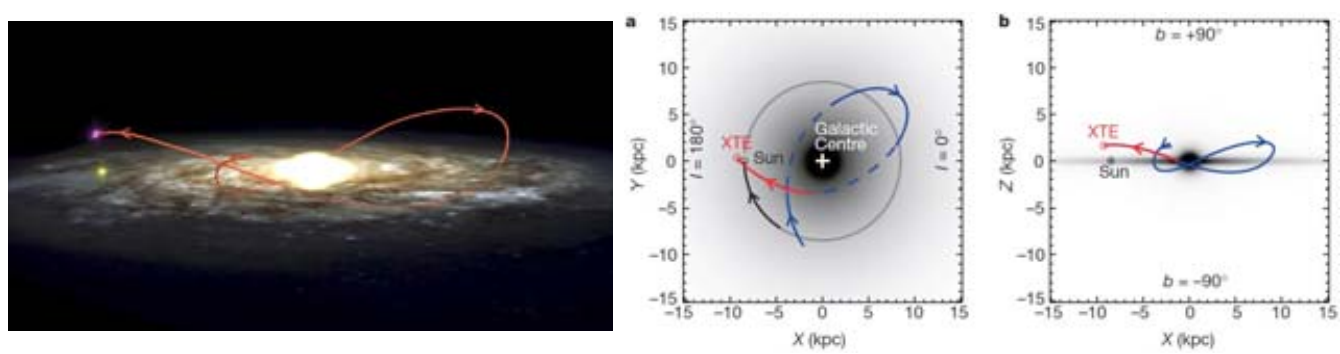

Figure 2. Galactic trajectory of a black hole wandering in the Galactic halo. This black hole may have been shoot out from the Galactic plane by an energetic, natal supernova explosion. Credit: Mirabel et al. (2001).

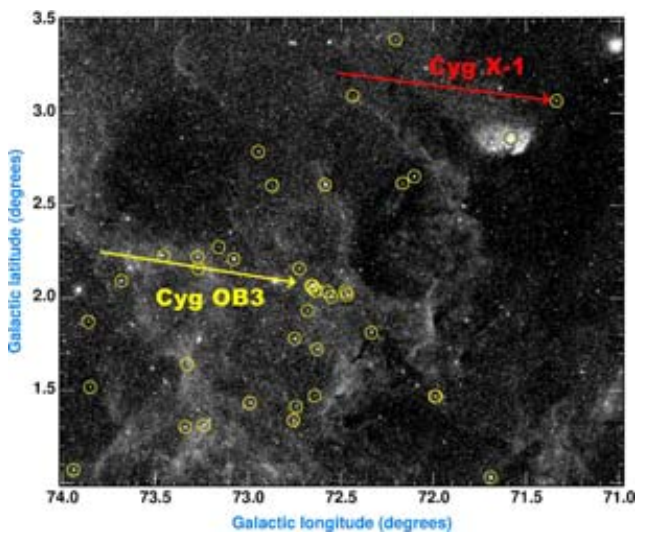

Figure 3. Kinematic evidence for the direct formation of the black hole in Cygnus X-1. Optical image of the sky around the black hole binary Cyg X-1 and the parent association of massive stars Cyg OB3. The red arrow shows the motion in the sky of the radio-counterpart of Cyg X-1 for the past 0.5 million years. The yellow arrow shows the average Hipparcos motion of the massive stars of Cyg OB3 (circled in yellow) for the past 0.5 million years. Cyg X-1 moves along with the parent association of massive stars indicating that the compact object did not received an energetic kick from a natal supernova. Credit: Mirabel \& Rodrígues (2003).

\section{The supermassive black hole at the Galactic Center}

Dynamics is the most direct method to determine the mass of astrophysical compact objects, and therefore, the best evidence for the existence of a black hole. The first unambiguous dynamic evidence for a supermassive black hole was found following the motion of water masers around the center of the galaxy NGC 4258.

More robust evidence has been obtained by the motion of stars around the dormant black hole of 3-4 million solar masses at the center of our Galaxy. These stars seem to be distributed in two randomly inclined disks of $0.04 \mathrm{pc}$ and $0.5 \mathrm{pc}$ radii. The unexpected discovery of a compact cluster of massive stars in the central parsec of our Galaxy (see figure 4) poses new questions and may open new horizons for our understanding of massive black hole formation and its relation with massive star formation. This central cluster exhibits a flat mass function and it is only 6 million years old. At present, it is not clear how it got there. Perhaps it was formed in situ. How this could take place under the strong tidal forces from the central black hole remains a mystery.

\section{Supermassive black holes in external galaxies}

Supermassive black holes are ubiquitous at the centers of galaxies. Their mass is correlated with the mass of the host galaxy, and in particular with that of the stellar bulge. This indicates that massive black hole and host galaxy formation are tided up.

The most massive black holes are found at $z \geqslant 6.0$ implying that they assembled very early, in less than one billon years after the Big Bang. On the other hand, supermassive black holes of lower mass have formed more slowly by merging at $\mathrm{z} \leqslant 3$. The peak 

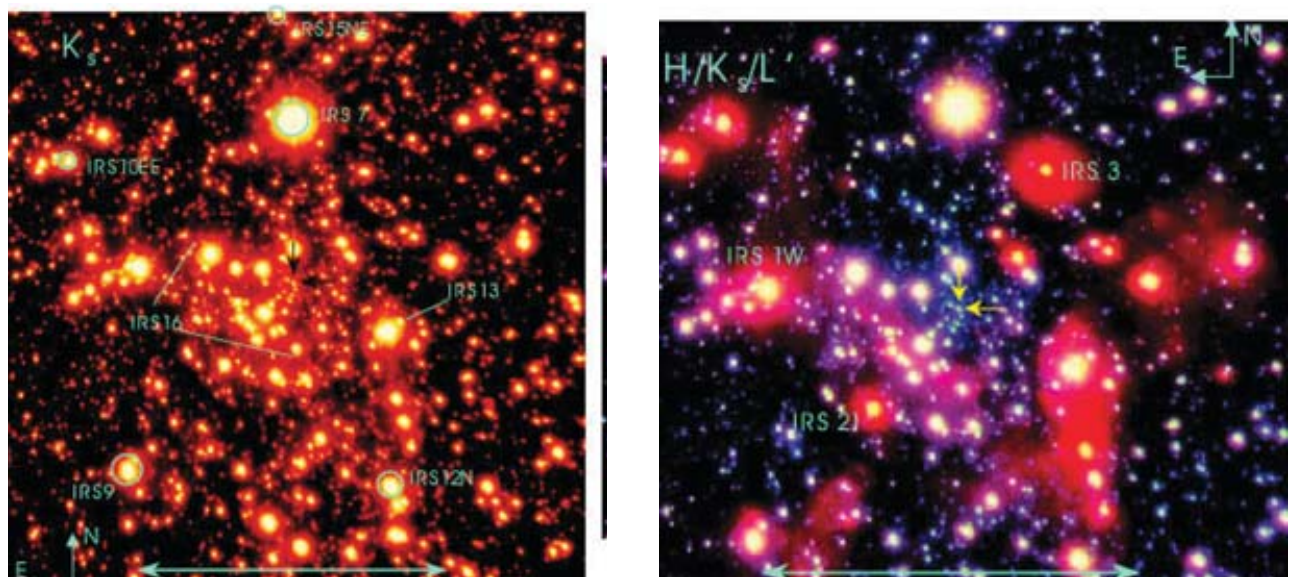

Figure 4. Left: The K-band near-infrared adaptive optics image of the central 20 arcsec of the Milky Way. Right: HKL colour composite image. Credit: Genzel et al. (2003).

accretion rate into black holes occurred rather late at $\mathrm{z} \sim 0.7$. Interestingly, this redshift is similar to the redshift of the peak density of luminous infrared galaxies at $\mathrm{z} \sim 0.8$, which suggests the association of maximum rate of accretion into black holes with dust enshrouded starbursts in luminous infrared galaxies, which are known to be mergers of gas-rich galaxies.

Although several models have been proposed, there is no general concensus on how supermassive black holes were form. More specifically, it is not known how could the most massive black holes in the universe form so rapidly with no feedback. Did supermassive black holes form before, after or coevally with the bulges?

The kick velocity due to gravitational recoil in merging supermassive black holes may displace the merged black hole from the dynamic center of the host galaxies. For dwarf galaxies the estimated kick velocity is larger than the typical escape velocities of 10-20 $\mathrm{km} / \mathrm{s}$, which may result in the ejection to the intergalactic medium of naked, massive black holes. How could we identify these runway black holes?

\section{Black holes of intermediate mass}

The existence of black holes of intermediate mass remains an open question. From the spectral properties of ultraluminous X-ray sources in nearby galaxies it has been suggested that some of these sources contain black holes of hundreds and perhaps thousands of solar masses. However, no dynamic evidence from the motion of satellite objects around such objects has been found. Furthermore, the luminosity function of black hole binaries as well as the properties of the most luminous x-ray black hole binaries in our Galaxy indicate that most of the super Eddington sources observed in nearby galaxies are a natural extension of the luminosity function of X-ray binaries, most of them being black holes with masses smaller than 100 solar. It has been argued that the hyperaccreting Galactic sources SS 433 and GRS 1915+105 seen from a different angle would be classified as ultraluminous X-ray sources.

If some ultraluminous x-ray sources are black holes of intermediate mass, one may ask why none has been identified in our Galaxy and/or the Magellanic Clouds, where the 
mass functions of compact objects can be determined. Black holes of intermediate mass may exist but are difficult to find.

\section{Correlations among black holes of all masses}

a) The fundamental plane: At low accretion rates, correlations between radio and x-ray luminosities are found for black holes across the whole range of masses. At low rates of accretion most the radiation power is dominated by synchrotron, compact, flat spectrum jets, from the x-rays to radio waves. For black holes in the jet dominated state, universal correlations between the x-ray luminosity, radio luminosity, and black hole mass have been found. Using this black hole fundamental plane it is expected that the masses of black holes could be determined from the x-ray and radio luminosities.

b) Accretion-jet coupling: Because of their proximity and rapid variability, microquasars have become the most adequate objects to study the connection between instabilities in the accretion disks and the genesis of relativistic jets. Figure 5 shows this connection as observed in an interval of time of a few tens of minutes. This sequence has also been observed in quasars, but on scales of a few years instead of tens of minutes.

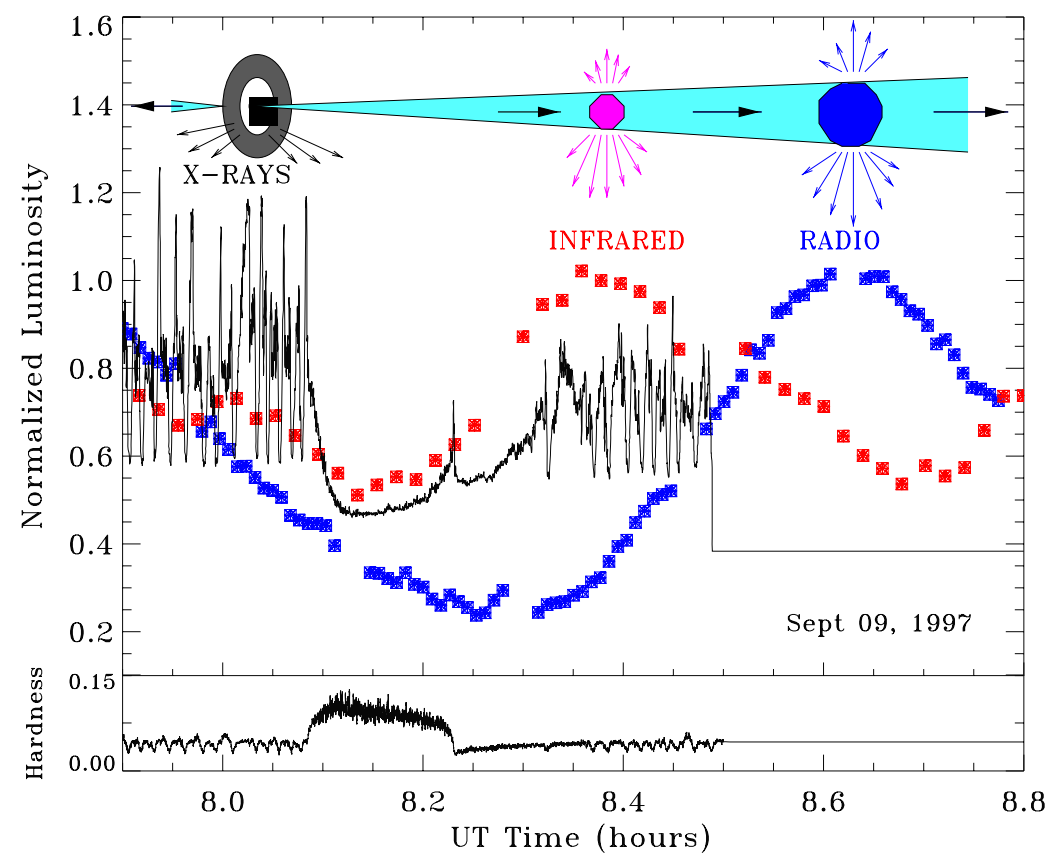

Figure 5. Real time observations in a microquasar of the connection between instabilities in the accretion disk and the genesis of jets in time scales of tens of minutes. Analogous disk-jet coupling has been observed in quasars, but on time scales of years. Credit: Mirabel et al. (1998).

c) Massive outflows: Across all mass scales, black holes in high luminous states exhibit X-ray absorption lines that reveal sub-relativistic winds with mass outflows as large as 0.3 times the accretion mass. Super-Eddington accretion makes massive outflows inevitable because all the accreting energy cannot be radiated.

In the case of black holes binaries the joint action of jets and massive outflows heat and blow away the interstellar medium, as does the black hole binary SS433 in the host nebula W50. X-ray observations with Chandra and XMM-Newton show that AGN produce analogous impact on the intergalactic medium, which has solved the long standing cooling flow paradox.

In this conference it was proposed that massive outflows may result from the interaction of relativistic jets with warped accretion disks, in both stellar and supermassive 
accreting black holes. Furthermore, it was proposed that bended disks may explain several intriguing observational results, such as the large numbers of obscured AGN, as well as the re-direction of precessing jets, as in SS 433. The following questions remain open:

Are the relativistic jets in AGN and microquasars purely leptonic or hadronic? Are the hadrons in the jets of SS433 a result of entrainment by the relativistic jets in the massive winds? Is the nebula W50 that hosts SS433 the remnant of the natal supernova of the compact object, or a blown away cavity by the super-winds, or both?

d) Quasi-periodic oscillations: Sgr A* accretes at low mass rates, and quasi-periodic, polarized flares on scales of tens of minutes have now been observed at X-rays, infrared, sub-millimeter and radio waves. At longer waves the flares are polarized up to $10 \%$. From the time lag at longer wavelengths it has been proposed that these flares could be synchrotron self Compton emission from adiabatically expanding clouds. It is unclear whether these expanding plasma clouds in Sgr $\mathrm{A}^{*}$ are rotating blobs in the accretion disk, or whether they are collimated expanding jets as observed in microquasars.

e) Spin: Black holes are the simplest objects in the universe. They are defined by only three parameters: the mass, the spin and the electric charge. Because much of the radiation emerges within 6 gravitational radii they provide a unique opportunity to probe gravity in the strong-field regime. The radius of the ultimate stable orbit depends from the spin, and knowing the mass, distance and inclination, it can be derived the spin by measuring the X-ray flux and the temperature of the accretion disk when the accretion disk is in the thermally dominated state and at luminosities $\leqslant 0.3$ Eddington.

Another way to measure the spin is with skewed fluorescence iron lines. Using both methods it has been claimed that some microquasars and AGN host extreme Kerr black holes. The following questions remain unanswered: Would magnetic fields change completely the physical conditions in the inner parts of accretion disks? Is there a general correlation between spin and jet power?

\section{Historical and epistemological analogy between stellar astrophysics and black hole astrophysics}

At present, black hole astrophysics is in an analogous situation as was stellar astrophysics in the first decades of the XX century. At that time, well before reaching the physical understanding of the interior of stars and the way by which they produce and radiate their energy, empirical correlations such as the HR diagram were found and used to derive fundamental properties of the stars, such as their mass. Analogous approaches are taking place in black hole astrophysics. Using correlations among observables such as the radiated fluxes in x-rays and radio waves, quasi-periodic oscillations, flickering frequencies, etc, fundamental parameters that describe astrophysical black holes such as the mass and spin of the black holes are being derived.

\section{References}

Genzel, R., Schödel R., Ott, T. et al. 2003, ApJ, 594, 812

Mirabel, I. F., Dhawan, V., Chaty, S. et al. 1998, A\&A, 330, L9

Mirabel, I. F., Dhawan, V., Mignani, R. P., Rodrigues, I. \& Guglielmetti, F. 2001, Nature, 413, 139

Mirabel, I. F. \& Rodríguez, L. F. 2002, Sky \& Telescope, 103 (May), 32

Mirabel, I. F. \& Rodríguez, L. F. 2003, Science, 300. 1119 


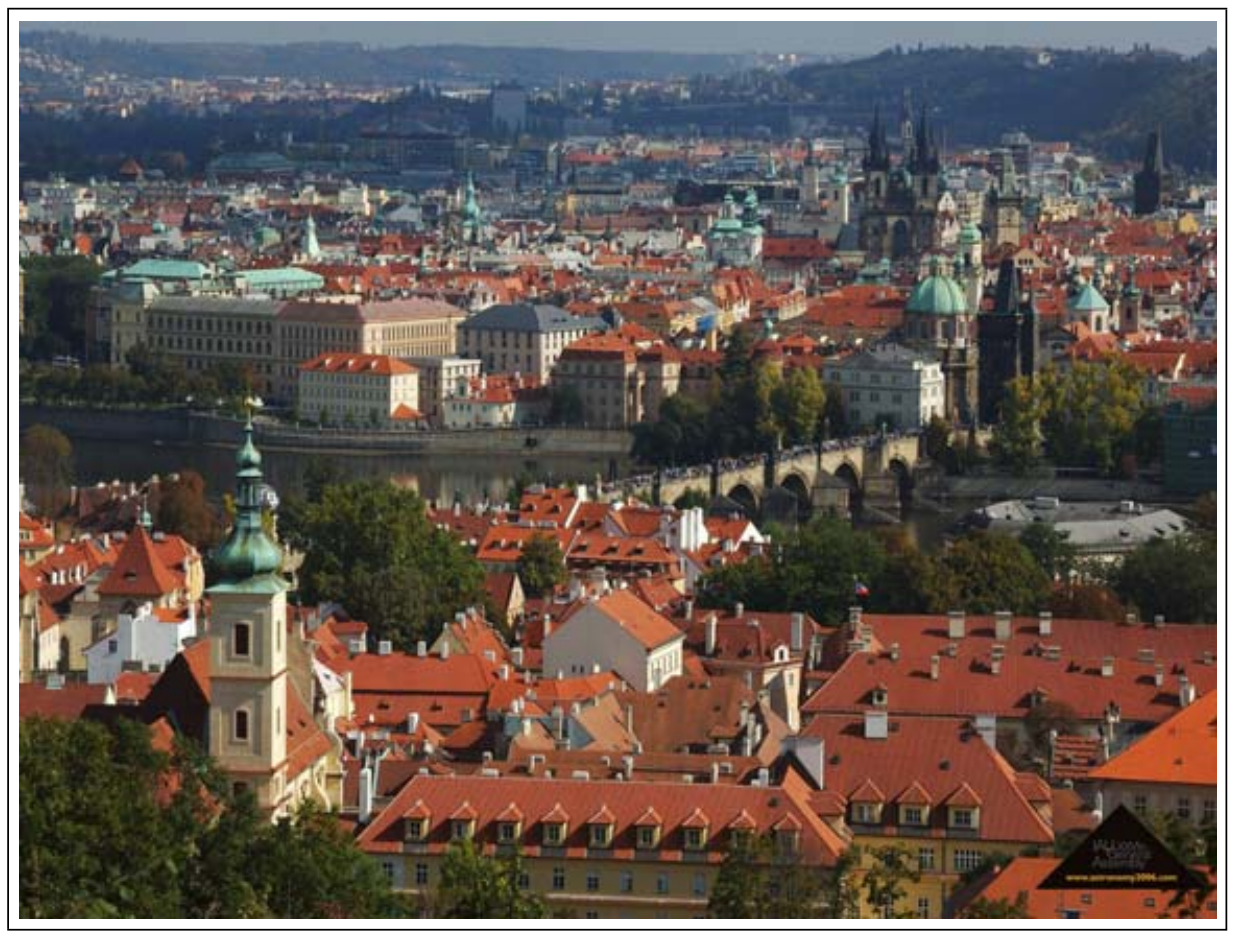

View of Prague 


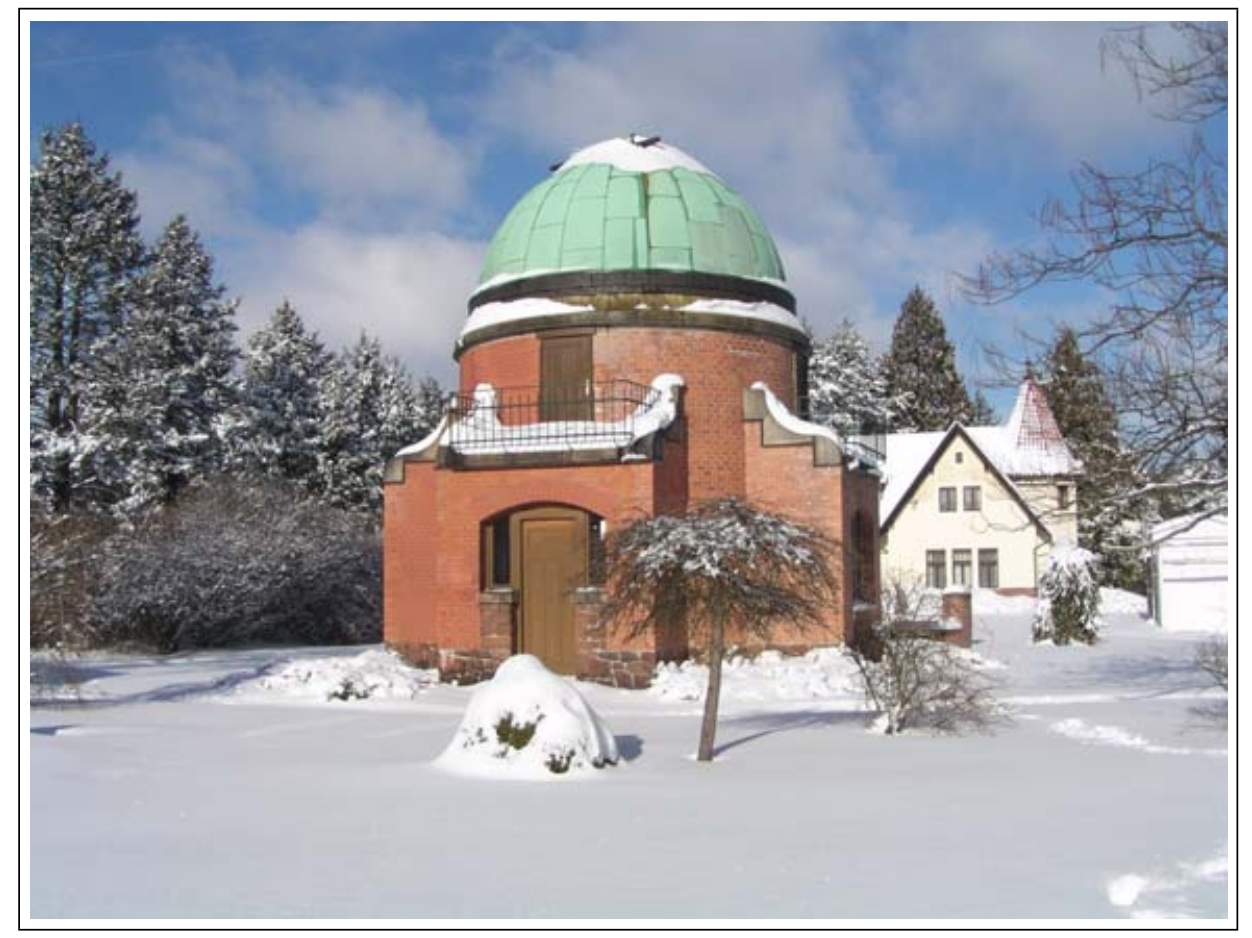

Historical dome of the Astronomical Institute in Ondřejov 